\title{
ESTREMA DESTRA E NEOPOPULISMO IN EUROPA
}

\author{
di Marco Tarchi
}

Hans-Georg Betz, Radical Right-Wing Populism in Western Europe, Houndmills-London, MacMillan, 1994, pp. 226, £ 12.99, Isbn 0-333-62809-8

LuCiano Cheles, Ronnie Ferguson e Michalina Vaughan (a cura di), The Far Right in Western and Eastern Europe, London-New York, 1995, pp. 378, £ 17.99, Isbn 0-582-23881-1

PETER H. MERKL e LEONARD WEINBERG (a cura di), The Revival of Right-Wing Extremism in the Nineties, London-Portland, Frank Cass, 1997, pp. 289, £ 16, Isbn 0-714-64207-X

Aurel Braun e STEPHEN SCHEINBERG (a cura di), The Extreme Right. Freedom and Security at Risk, Boulder, Westview Press, 1997, pp. 293, £ 17.50, Isbn 0-8133-3151-X.

Le ragioni che hanno determinato uno status molto incerto nel lessico delle scienze sociali del concetto di «estrema destra», e che spingono ad usare la nozione con cautela, sono molteplici e, almeno in parte, certamente fondate. È comprensibile, ad esempio, la diffidenza con cui molti studiosi guardano all'uso delle metafore spaziali nell'analisi della politica, dal momento che l'annosa controversia sulla capacità della più celebre di esse - la diade dicotomica destra/sinistra - di offrire una rappresentazione soddisfacente delle linee di conflitto politico e ideologico che attraversano le società contemporanee è tuttora viva ${ }^{1}$, $\mathrm{e}$ lontana dal trovare soluzione. Né si può sottovalutare il rischio

1 Anche una semplice ricapitolazione del dibattito sul significato e l'utilità di queste categorie occuperebbe troppo spazio. $\mathrm{Ci}$ limitiamo pertanto a segnalare alcuni dei documenti più significativi: Laponce (1981), Cofrancesco (1984), Galeotti (1984), Gauchet (1994), Bobbio (1995), Campi e Santambrogio (1997) e, in chiave meno teorica, Inglehart e Klingemann (1976). Il recente volume di Santambrogio (1998) offre una sintetica panoramica della discussione. Per le nostre opinioni in argomento, rinviamo a Tarchi (1994).

RIVISTA ITALIANA DI SCIENZA POLITICA / a. XXVIII, n. 2, agosto 1998 
che, maneggiando un'espressione inflazionata dagli usi del gergo giornalistico e solitamente impiegata con intenzioni svalutative si finisca, anche senza volerlo, con l'inoltrarsi sul terreno dei giudizi di valore. Resta il fatto però che, in questo come in altri casi - basti pensare alle alterne sorti del termine «totalitarismo» ${ }^{2}-$, i dubbi sulla plausibilità dell'etichetta con la quale si denomina un fenomeno non annullano la specificità del fenomeno stesso e devono tradursi non in una sospensione delle indagini nel campo ma in uno sforzo di reinquadramento e ridefinizione dell'oggetto di studio.

Ciò è oggi particolarmente necessario per due ordini di motivi. Da un lato, perché la scarsità e la non eccelsa qualità delle ricerche prodotte sino a pochi anni addietro sull'argomento che qui ci interessa è stata il frutto di riserve psicologiche e morali sull'opportunità di descrivere e capire un aspetto «riprovevole» della realtà politica che non possono essere condivise da una scienza politica che faccia propria la lezione weberiana dell'avalutatività. Dall'altro, perché negli anni ottanta l'espressione «estrema destra» è stata da varie parti adottata per definire una delle innovazioni più significative della scena politica europea: la comparsa di una nuova famiglia di partiti (i più noti dei quali sono il Front national francese, i Republikaner tedeschi e la Fpö austriaca) che, in simmetria con le formazioni ecologiste nate sulla scia dei Grünen, ha messo in crisi lo schema dei rapporti tra conflitti sociali e sistemi partitici tratteggiato da Stein Rokkan nella sua ormai classica teoria dei cleavages. La sospensione del tabù vigente su questa scottante materia, e la conseguente fioritura di opere di impianto politologico concepite allo scopo di scandagliarla, va dunque accolta con soddisfazione, a patto di riuscire a orizzontarsi fra le numerose proposte di revisione terminologica e contenutistica che esse avanzano, con effetti non sempre chiarificatori.

A giudicare dai risultati del lavoro svolto dai collaboratori di diversi progetti di ricerca, contenuti nei quattro volumi di recente pubblicazione di cui ci occupiamo ${ }^{3}$, molto cammino resta,

2 Sul punto, rinviamo a Tarchi (1997).

$3 \mathrm{La}$ produzione in argomento dell'ultimo decennio comprende molti altri titoli, di valore diseguale. Per limitarci ai lavori concepiti in un'ottica comparativa, e tralasciando quelli ispirati esclusivamente da preoccupazioni «militanti», segnaliamo Harris (1990), Hainsworth (1992), Merkl e Weinberg (1993), Ignazi (1994), Bjørgo (1995), oltre alla prima edizione di Cheles, Ferguson e Vaughan (1991). 
in questa direzione, da percorrere. Manca ancora un consenso unanime sull'etichetta da applicare al soggetto sotto osservazione - oltre che di «estrema destra», termine scomposto nelle versioni angloamericane in extreme right, far right, right-wing extremism, si parla di «destra radicale», di «neofascismo», di «radicalismo populista di destra», di «populismo» tout court, di «nuova destra»-, sull'individuazione delle unità da assegnare alla categoria (c'è chi propone di includervi la Lega Nord e il partito degli automobilisti svizzero, chi dubita di poter mettere sullo stesso piano la Fpö di Jörg Haider e i gruppi neonazisti, chi amplia il concetto ai nazionalconservatori di Philippe de Villiers e chi invece alle bande skinbeads) e sui suoi contenuti definitorii. Tuttavia, qualche pista per orizzontarsi nel magmatico campo è ormai stata tracciata, e a partire da essa possono essere raccolte un certo numero di osservazioni di ordine generale utili a prevedere le possibili evoluzioni del panorama politico, soprattutto europeo, dei prossimi decenni.

Un primo punto fermo riguarda la rilevanza e l'estensione del fenomeno. Diversamente da quanto era accaduto in passato, quando gli exploits elettorali di liste ispirate a ideologie neofasciste (Drp, Srp e Npd nella Repubblica federale tedesca, Msi in Italia) erano rimasti episodi isolati e legati ai rispettivi contesti nazionali, tanto da far parlare del basso potenziale di sostegno dell'estremismo di destra come di una "normale" condizione patologica» delle democrazie industrializzate ${ }^{4}$, l'odierna ascesa dei partiti «radical-populisti» pare destinata a consolidarsi sia sul piano elettorale che su quello organizzativo e ad allargare progressivamente il proprio raggio di azione. Anche lasciando da parte la manifestazione di tendenze analoghe in paesi collocati in altri ambiti geopolitici, come il Nord America o il Sudafrica, è infatti ormai evidente che non solo in buona parte dell'Europa occidentale ma anche nella quasi totalità dei regimi dell'Est postcomunista (fra i casi esaminati dagli autori compresi in questa rassegna figurano Russia, Ungheria, Polonia, Romania, Ucraina, Bielorussia, Slovacchia, Repubblica Ceca ed ex Jugoslavia) la presenza del nuovo ceppo politico non è più un dato occasionale. Ad avviso di Hans-Georg Betz, addirittura, la destra radicale «per sfida programmatica e potenziale elettorale rappresenta il fenomeno politico potenzialmente più dinamico e

4 Cfr. Scheuch e Klingemann (1967). 
dirompente degli anni novanta», segno di trasformazioni politiche e socio-economiche fondamentali; e il giudizio è sostanzialmente sottoscritto, pur con minore enfasi, da altri analisti.

Un secondo convincimento che pare accomunare i ricercatori è che l'attuale ondata di radicalismo di destra non si configura come una mera reviviscenza nostalgica, ma piuttosto come il prodotto di tensioni inedite, estranee ai punti di frattura della storia europea degli ultimi due secoli. L'ammissione della relativa originalità del fenomeno non si traduce però in un pieno accordo sulla sua distanza rispetto ai modelli del passato, come si può agevolmente constatare passando dall'uno all'altro dei libri che ci servono da guida in questa esplorazione. Il reader curato $\mathrm{da}$ Cheles, Ferguson e Vaughan insiste ad esempio più degli altri sugli aspetti di continuità che legherebbero comunque l'attuale estrema destra a quella che agì da protagonista negli anni fra le due guerre mondiali (non a caso, la prima versione dell'opera, che non comprendeva i capitoli sull'Est Europa, si intitolava Neo-Fascism in Europe e prendeva in considerazione in primo luogo paesi in cui vi erano stati periodi di governo fascista o «fascisteggiante»). Pur offrendo una panoramica dettagliata dello sviluppo delle correnti neoautoritarie, populiste e xenofobe del vecchio continente, con incursioni sul terreno delle radici culturali (Douglas Johnson sulla nouvelle droite) e dell'immagine (Luciano Cheles con un ampio saggio sulla propaganda del Msi, sospesa fra tradizione e innovazione), il libro insiste su questa impostazione con effetti a volte sconcertanti, ben illustrati dal saggio introduttivo di Jaroslav Krejči, dove si legge che «politiche che possono essere descritte come neofasciste» si riscontrano oggi a Cipro, in Bosnia e nell'Ulster e pochi anni orsono erano perseguite, «senza vergogna», dai regimi comunisti rumeno e bulgaro. E difficile non vedere, in queste parole, un esempio di quello che Michi Ebata, nel volume curato da Braun e Scheinberg, chiama «il pericolo di usare il concetto [di estrema destra] come un'arma politica per screditare gli avversari», e non rilevarne la distanza dall'approccio di Peter Merkl, il quale, dopo aver messo in guardia dal «cattivo uso delle vecchie etichette», giudica la riemersione di movimenti e attività della destra radicale «in larga misura nuova e sui generis» e ritiene che essa vada indagata come un fenomeno di nuovo conio, causato da condizioni e circostanze recenti. Il lamento di Merkl, secondo cui «i fantasmi dell'era fascista di mezzo secolo e più fa continuano a sfuocare la nostra analisi dei fenomeni del più recente passato», 
è certamente appropriato all'etica scientifica, ma non sembra trovare molto ascolto, se nel suo recentissimo contributo Stephen Scheinberg, tirando le somme degli scritti dei suoi collaboratori, deduce dai successi di Zhirinovskij o di Le Pen che «il fascismo è diventato di nuovo politicamente rispettabile» e qualifica Fini come il leader del fascismo italiano.

Da queste palesi contraddizioni trae una paradossale conferma un terzo rilievo che, sia pure soltanto in negativo, funge da filo rosso di molti degli interventi compresi nei quattro volumi: le tradizionali interpretazioni dell'estremismo di destra non sono adeguate a spiegarne le odierne evoluzioni, ed è dunque giunta l'ora di mettere mano a una nuova definizione. Ma è proprio la consapevolezza diffusa di questa carenza a mostrare come gli studi nel campo non siano ancora usciti dall'impasse.

Il saggio di Michi Ebata, Right-Wing Extremism: In Search of a Definition (Braun e Scheinberg 1997, 12-35) è lo specchio più fedele del dilemma. Accogliendo indirettamente il suggerimento di Giovanni Sartori che già quindici anni fa invitava a trattare i concetti di destra e di sinistra, per farne buon uso, in modo convenzionale e relativo ${ }^{5}$, Ebata sostiene che, essendo una definizione essenzialistica dell'estrema destra impossibile, occorre trovarne una nominale, e che, per raggiungere lo scopo, è opportuno passare da una delineazione spaziale-statica della nozione, troppo dipendente da un idealtipo, a una «dinamica e temporale». Il perseguimento di questo obiettivo lo porta a criticare i tentativi di spiegazione monocausali, nonché quelli offerti dalle teorie psicosociali (dall'indagine di Adorno sulla personalità autoritaria in poi) e socioeconomiche (ben illustrate da Lipset e Raab 1970). Ma quando si tratta di declinare gli esiti di questa revisione, gli elementi di novità latitano: i temi accomunanti dell'estremismo di destra sono per Ebata l'astio, il fanatismo, il nazionalismo etnocentrico, il pregiudizio basato su un'ideologia della diseguaglianza e dell'esclusione, e i suoi scopi si riducono all'eliminazione dello stato democratico moderno.

Più promettenti sono i percorsi di analisi proposti da alcuni

5 Il riferimento è a Sartori $(1982,255-256)$, dove si legge che destra e sinistra sono immagini spaziali «sprovviste di "ancoraggio semantico"», «contenitori vuoti aperti a tutti i travasi, a tutti i contenuti», che «stanno per pacchetti di issues, per una serie di prese di posizione su una serie di questioni controverse» solo se assunte «in ogni singolo tempo, momento o periodo storico». Il ragionamento è concepito per destra e sinistra, ma lo si può applicare, a maggior ragione, ai loro derivati. 
dei coautori di The Revival of Right-Wing Extremism in the Nineties: Peter Merkl, Piero Ignazi e Michael Minkenberg.

Merkl sottolinea la necessità di abbandonare la ricerca di una «"quasi platonica" essenza del fascismo» per orientarsi verso una definizione situazionale basata sugli atteggiamenti concretamente assunti dai partiti di estrema destra sulle varie issues in discussione. Consapevole della difficoltà di giungere a questo risultato, dal momento che questi partiti possono trovarsi, su una serie di problemi, a convergere con forze più moderate o conservatrici, egli presta comunque attenzione ai tentativi di ricavare dall'indagine empirica una definizione sostantiva, limitando i dati qualificanti su questo terreno a tre: la memoria nostalgica dell'egemonia fascista, la reazione ai valori e agli stili di vita proposti dalla generazione del ' 68 e il rifiuto degli immigrati.

L'impostazione che Ignazi dà alla questione è più articolata. Per studiare le somiglianze che autorizzano a parlare di una nuova famiglia di partito attiva in numerosi paesi europei, suggerisce un approccio parsimonioso: lasciare da parte lo studio delle strutture organizzative, dello stile della leadership e delle caratteristiche dell'elettorato per concentrarsi sull'ideologia, collegandola alla collocazione all'interno dello spazio competitivo e all'atteggiamento verso il sistema. Applicando tale schema, Ignazi giunge a definire l'estrema destra come il soggetto politico che fa propria l'ideologia del fascismo - intesa come «una forma palingenetica di ultranazionalismo populista», secondo la lettura proposta dallo storico britannico Roger Griffin -, occupa «lo spazio politico-ideologico dove il fascismo è il riferimento basilare», collocato alla destra del raggio di azione dei partiti conservatori, e assume atteggiamenti di opposizione al sistema democratico, espressi sotto forma di antiparlamentarismo, antipluralismo e antipartitismo. La famiglia così identificata è poi suddivisa in due varianti: l'estrema destra tradizionale, che è stata incarnata tipicamente dal Msi, e quella post-industriale, di cui è portabandiera il Front national. Questa seconda variante, oggi nettamente predominante, non è un neofascismo mascherato, bensì il frutto di nuovi conflitti, non più centrati solo attorno $a$ interessi materiali ma estesi all'intero spettro dei valori «postmaterialisti» insinuatisi nelle società europee dopo il 1968.

Pur distaccandosene nella terminologia, Minkenberg condivide con Ignazi la convinzione che i «partiti della nuova destra» (come preferisce chiamarli) rappresentino una risposta al post- 
materialismo della nuova sinistra, sostenendo un'ideologia che $\mathrm{fa} \mathrm{da}$ «controparte dialettica della modernizzazione politica». Più che a una reazione di circostanza connessa a singoli problemi come la crescita dell'immigrazione extraeuropea, la loro presenza va collegata, a suo avviso, all'emersione di un vero e proprio contromovimento, attivo su due dimensioni: intellettuale (come fucina internazionale di idee che traggono spunto soprattutto dagli autori della Rivoluzione conservatrice tedesca degli anni venti e trenta) e politica (come radicalismo conservatore neopopulista). Istigati più dallo «sciovinismo del benessere» che da nostalgie autoritarie, il loro atteggiamento non può dirsi antisistemico in senso proprio: assumono posizioni estreme, ma sempre collocate all'interno dell'ordine costituzionale, operano ideologicamente lungo il medesimo asse politico dell'estrema destra di qualche decennio addietro ma ne ammorbidiscono le rivendicazioni per introdurle nell'agenda politica ufficiale, fungendo da cerniera, ma anche da linea di separazione, fra i settori dell'opinione pubblica moderata resi più inquieti dalla caduta del vecchio ordine morale e sociale e gli ambienti dell'estremismo antidemocratico.

Chi appare maggiormente convinto della non riducibilità della destra radicale di questo ultimo scorcio di secolo ai canoni classici dell'estrema destra filofascista è Hans-Georg Betz. Il suo libro descrive una famiglia politica relativamente omogenea, contraddistinta da una comune insistenza programmatica sulla lotta all'immigrazione (non solo extraeuropea, ma anche in provenienza dai paesi ex-comunisti) e all'insicurezza, sulla valorizzazione dell'identità nazionale e sulla denuncia dei mali causati dalla «partitocrazia», ma anche da posizioni liberali in ambito sociale ed economico. Radicali nell'opposizione al sistema e alle sue basi socioculturali e sociopolitiche, i partiti che si collocano entro questa corrente vogliono un libero mercato e una minore invadenza dello stato nella sfera delle attività private, e proclamano il diritto alla realizzazione individuale, reclamando dalla mano pubblica soltanto la tutela dell'ordine e della sicurezza. Di destra in quanto rifiutano l'egualitarismo e l'integrazione sociale dei gruppi marginali, spingendosi spesso sul terreno dell'aperta xenofobia, sono populisti nell'appello all'uomo comune e al suo «buonsenso» e strumentalizzano i sentimenti di ansia e delusione nei confronti dell'establishment diffusi in taluni settori sociali. Nati come strumenti di reazione difensiva alle conseguenze dell'erosione delle subculture, delle istituzioni e degli 
ambiti che sorreggevano le identità collettive tradizionali, traggono alimento dal risentimento e dall'alienazione che investono $i$ ceti più negativamente toccati dalla globalizzazione, il che spiega la graduale ma consistente proletarizzazione del loro elettorato (il Front national e la Lega Nord - che Betz include nella sua analisi assieme a Fpö, Republikaner, Vlaams Blok e «partiti del progresso» scandinavi - sono ormai i partiti più votati dall'elettorato operaio in Francia e nell'Italia del Nord). Definirli neofascisti non ha alcun fondamento; anzi, la stessa etichetta populista può non essere più sufficiente a rendere ragione della loro varietà ed esige una specificazione, che distingua tra partiti «populisti liberali» (le formazioni antitasse del Nord e Centro Europa) e «nazionalpopulisti».

Le opinioni sullo schema di classificazione da adottare per poter considerare ed esaminare unitariamente questi nuovi attori della politica europea sono dunque assai varie. Molto minori sono invece le divergenze quando si tratta di individuare le ragioni dei successi elettorali che essi vanno oggi riscuotendo. Come l'articolo di Lauri Karvonen The New Extreme RightWingers in Western Europe: Attitudes, World Views and Social Characteristics (Merkl e Weinberg 1997, 91-10), basato sui dati di sondaggio dell'European Value Study, ben argomenta, alcuni atteggiamenti tradizionalmente correlati all'estremismo di destra - culto dell'autorità, intolleranza verso la «diversità», antisemitismo - sono in netto calo un po' in tutta Europa, ma crescono altri sentimenti che possono fare da terreno di coltura del nuovo populismo: ostilità verso gli stranieri, orgoglio nazionale, preoccupazioni per la sicurezza.

E su questa miscela di timori e aspirazioni che i partiti della «destra radicale» possono capitalizzare, espandendo la propria nicchia di mercato elettorale. Nell'Europa orientale, almeno nell'immediato futuro, un veicolo di penetrazione è offerto dal nazionalismo dei movimenti neopopulisti, alla cui base, come suggerisce Anthony D. Smith (The Dark Side of Nationalism: The Revival of Nationalism in Late Twentieth-century Europe, in Cheles, Ferguson e Vaughan 1995, 13-19), vi è un'idea di nazione non «civica e territoriale» ma «etnica e genealogica», capace di attivare una mobilitazione vernacolare che, politicizzando la cultura, può suscitare consensi attorno all'idea di «purificare» da presenze aliene la cultura e la comunità nazionale. Nei paesi occidentali, dove l'irredentismo non ha praticamente più presa anche per effetto del processo di unificazione continentale, la 
questione-immigrazione, che sembra sempre più espandere i suoi effetti sin quasi a configurare un nuovo cleavage, è invece il perno centrale della penetrazione delle formazioni della destra radicale in ambienti sociali dove neppure i movimenti fascisti, nell'epoca di maggior successo, erano riusciti a trovare buona accoglienza. Sbaglierebbe però chi individuasse nel lepenismo e nei suoi partiti-fratelli una sorta di single-issue movement; è il collegamento della protesta anti-immigrati, cavalcata sempre meno occasionalmente anche da forze politiche conservatrici ben più «rispettabili», con altri temi forti (difesa dell'identità nazionale, tutela della sicurezza individuale e collettiva, protezionismo economico, ribellione contro il fisco, ecc.) a consentire al neopopulismo di coltivare una porzione di spazio politico autonoma e difficilmente insidiabile dai concorrenti. Le analisi di Betz, ed altre che le hanno precedute e seguite, dimostrano che il voto per l'estrema destra «post-industriale» è in primo luogo un voto contro le disfunzioni dei sistemi politici democratici, che proviene da ambiti di estrazione politica molto diversa, tanto da far configurare i partiti che ne beneficiano come «partiti pigliatutto di protesta».

Sovente descritta come qualunquistica, questa protesta sembra invece aver individuato alcuni precisi bersagli ed appoggiarsi su un retroterra di principii - produttivismo, enfatizzazione dell'efficienza e dell'ordine, attaccamento al locale contro il globale - che attraversano le linee di antagonismo politico ed elettorale consolidatesi negli anni della Guerra Fredda e del secondo dopoguerra e possono offrire a chi se ne fa interprete un solido zoccolo di consenso (sta a dimostrarlo la tendenza al consolidamento e/o alla crescita della Fpö, del Front national e della Lega Nord, che si rafforza di elezione in elezione malgrado lo scarso potenziale di coalizione di cui questi partiti dispongono). È probabile che, come è ancora Betz a sostenere, votino per le liste populiste soprattutto gli «abbandonati» dal processo di trasformazione post-industriale del capitalismo o, per dirla con Merkl, molti di coloro che sono rimasti esclusi dalla «rivoluzione comunicativa» che si è accompagnata alla crescita della «società dei due terzi»; ma non è affatto detto che, col progredire della globalizzazione, queste categorie di insoddisfatti siano destinate a restringersi numericamente. È piuttosto probabile il contrario. Se così dovesse essere, gli studiosi della politica dovranno rassegnarsi a constatare di trovarsi di fronte non ad un altro effimero remake del poujadismo ma ad un nuovo attore, 
relativamente stabile, della competizione democratica. E dovranno attrezzarsi a capirne e valutarne le caratteristiche e i comportamenti non attraverso gli esorcismi e il ricorso alle metafore mediche sulle «diagnosi» e le «cure» che ancora abbondano nella letteratura in argomento, e che sono adatte semmai a fronteggiare eventi patologici, quanto piuttosto con gli usuali strumenti della ricerca scientifica: gli unici che consentono di aggiornare utilmente e in modo appropriato la conoscenza fisiologica dei sistemi politici.

\section{Riferimenti bibliografici}

Bjørgo, T. (a cura di) (1995), Terror from the Extreme Right, London, Frank Cass.

Bobbio, N. (1995), Destra e sinistra. Ragioni e significati di una distinzione politica, nuova ed. riveduta ed ampliata, Roma, Donzelli.

Campi, A. e A. Santambrogio (a cura di) (1997), Destra/Sinistra. Storia e fenomenologia di una dicotomia politica, Roma, Pellicani.

Cheles, L., R. Ferguson e M. Vaughan (a cura di) (1991), Neo-Fascism in Europe, London-New York, Longman.

Cofrancesco, D. (1984), Destra e sinistra. Per un uso critico di due termini chiave, Verona, Bertani.

Galeotti, A.E. (1984), L'opposizione destra-sinistra. Riflessioni analitiche, in F. Ferraresi (a cura di), La destra radicale, Milano, Feltrinelli, pp. 253-275.

Gauchet, M. (1994), Storia di una dicotomia. La destra e la sinistra, Milano, Anabasi.

Hainsworth, P. (a cura di) (1992), The Extreme Right in Europe and the USA, London, Pinter.

Harris, G. (1990), The Dark Side of Europe. The Extreme Right Today, Edinburgh, Edinburgh University Press.

Ignazi, P. (1994), L'estrema destra in Europa, Bologna, Il Mulino.

Inglehart, R. e H.D. Klingemann (1976), Party Identification, Ideological Preference and the Left-Right Dimension Among Western Mass Publics, in I. Budge, I. Crewe e D. Farlie (a cura di), Party Identification and Beyond, New York, Wiley, pp. 243-273.

Laponce, J.A. (1981), Left and Right. The Topography of Political Perceptions, Toronto, University of Toronto Press.

Lipset, S.M. e E. Raab (1970), The Politics of Unreason: Right-Wing Extremism in America, 1790-1970, New York, Harper \& Row.

Merkl, P. e L. Weinberg (a cura di) (1993), Encounters with the Contemporary Radical Right, Boulder-San Francisco-Oxford, Westview Press. 
Santambrogio, A. (1998), Destra e sinistra. Un'analisi sociologica, Roma-Bari, Laterza.

Sartori, G. (1982), Teoria dei partiti e caso italiano, Milano, SugarCo.

Scheuch, E.K. e H.D. Klingemann (1967), Theorie des Rechtsradikalismus in westlichen Industriegesellschaften, in «Hamburger Jahrbuch für Wirtschafts- und Gesellschaftspolitik», vol. 12, pp. 11-29.

Tarchi, M. (1994), Destra e sinistra: due essenze introvabili, in «Democrazia e Diritto», XXXIV, 1, pp. 381-396.

- (1997), Il totalitarismo nel dibattito politologico, in «Filosofia politica», XI, 1, pp. 63-79. 